\title{
Altered DNA methylation associated with nervosa anorexia in males
}

\author{
Artem Kim ${ }^{1}$, Brigitte Izac ${ }^{2}$, Nicolas Lebrun ${ }^{3}$, Nicolas Ramoz ${ }^{3}$, Corinne Blanchet ${ }^{4}$, Franck Letourneur ${ }^{2}$, Marie Rose Moro ${ }^{4}$, Philip Gorwood ${ }^{3,5}$, \\ Marie De Tayrac ${ }^{1}$ and Thierry Bienvenu ${ }^{3,6 *}$ \\ ${ }^{1}$ Institute of Genetics and Development of Rennes (Igdr), Rennes, France \\ ${ }^{2}$ Cochin Institute, Genomics Platform, Inserm U1016, Paris, France \\ ${ }^{3}$ Institute of Psychiatry and Neuroscience of Paris (IPNP), Inserm U1266, Paris, France \\ ${ }^{4}$ Teenagers' House, Cochin Hospital, Paris, France \\ ${ }^{5} \mathrm{CMME}$, St. Anne's Hospital, University Paris-Descartes, France. \\ ${ }^{6}$ Laboratory of Molecular Genetics and Biology, Hôpital Cochin, HUPC, AP-HP, Paris, France
}

\begin{abstract}
Purpose: Anorexia nervosa (AN) is a serious psychiatric disorder characterized by abnormal eating behaviors, resulting in weight loss and increased mortality. Although more common in females, an estimated 5 to $10 \%$ of affected patients are males. Up to now, the exact cause of male AN is unknown. As with many psychiatric diseases, it's probably a combination of genetic, biological, psychological and environmental factors. Here, we used whole-genome bisulfite sequencing to determine the methylome of male individuals with AN.
\end{abstract}

Methods: We analyzed by bisulfite sequencing 3,340,894 biologically relevant CpG sites (Illumina TruSeqMethyl Capture EPIC kit) of 6 male patients affected with AN restrictive type. To reduce the environment effect, 4 related unaffected individuals were selected as controls.

Results: Comparisons between male patients affected with AN restrictive type and unaffected controls showed 153 differentially methylated regions and 1812 differentially methylated $\mathrm{CpGs}$ that corresponded to genes relevant to metabolic and nutritional status, psychiatric status and immune function. Moreover, the String network analysis software identified a subnetwork, related to MAPK signaling pathway, PI3K-Akt signaling pathway and neurotrophin signaling pathway.

Conclusions: Our findings replicate several results concerning several target genes such as PRKAG2, RPTOR, and ICAM5 previously identified in female AN, and identified novel signaling pathways involving PI3K-Akt and neurotrophin signaling pathway disturbed in AN.

\section{Introduction}

Anorexia nervosa (AN) is a complex neuropsychiatric disorder characterized by weight loss, and an intense fear of gaining weight. Family and twin studies of AN have shown that genetic and environmental factors play important roles in the pathogenesis of AN. Twin studies have estimated the heritability to be $56 \%$ [1]. However, while several genes were identified by candidate gene studies and genome-wide association studies (GWAS), they often failed to replicate in other studies [2-5]. The same observation was found for major depression sharing common genetic and environmental risk factors with AN [6]. Indeed, young people who suffered from AN are at high risk of depression [7]. Epigenetics and environmental factors might play a crucial role in the development of $\mathrm{AN}$ as well as in major depressive disorder [8]. Recently, a genome-wide DNA methylation study was conducted in women with active $\mathrm{AN}, \mathrm{AN}$ in remission and in non-AN controls. Global analysis of the 3 groups revealed 295 differentially methylated sites (DMS) representing 277 genes. Some of the identified genes were related to nutrition, to bone and tissue heath, immune function/inflammation, as well as general or transcriptional processes, and glia-neuron interaction [9].

As $\mathrm{AN}$ is nine times more often in females than in males, focusing on gender specificities might help to shed light on its overall nature. We therefore propose an analysis of several male AN patients, supporting in part the recent findings and identifying a novel disease-relevant pathway.

\section{Patients and methods}

We analyzed more than 3.3 M biologically relevant CpGs (Illumina TruSeqMethyl Capture EPIC kit) of 6 male patients affected with AN restrictive type. This approach captures 3,340,894 CpG sites including 26,981 CpG islands (107 Mb), including American College of Medical Genetics (ACMG) genes, coding genes known to be involved in cancer, coding exons from Ensemble 70, and 100 promoters defined as being of high interest and difficult to sequence. To reduce the environment effect, 4 related unaffected individuals- parents of two AN individuals - were selected as controls. Briefly, libraries were prepared according to manufacturer protocol (using the TrueSeq DNA Methylation Kit (cat. EGMK81312, Illumina Inc., San Diego, CA), the NEXTflex

*Correspondence to: Thierry Bienvenu, Institute of Psychiatry and Neuroscience of Paris, 102 rue de la Santé, 75014 Paris, France, E-mail thierry.bienvenu@inserm.fr

Key words: anorexia nervosa, restrictive type, anorexia, whole-genome bisulfite sequencing

Received: July 12, 2019; Accepted: July 22, 2019; Published: July 30, 2019 
Bisulfite Sequencing Kit (cat. 5119-02) and the 24 NEXTflex Bisulfite Sequencing Barcodes (cat. 511913)). After PCR-amplification, the bisulfite-treated Libraries were clustered on a V3 paired-end read flow cell and sequenced for 100 cycles on an Illumina NextSeq500 System (Illumina). Fastq files were generated using Illumina's software CASAVA v1.8.2. Raw fastq reads were processed by a custom pipeline that consists of: (i) filtering raw fastq reads for pass filter reads, (ii) trimming adapter sequence by Trim Galore, (iii) genomic alignments performed using Bismark to reference human genome hg19, and (iv) methylation calling by a Bismark script (https://github.com/nfcore/methylseq). The $\mathrm{R}$ package methyKit was used for analysis and annotation of DNA [10]. After adjustment for potential confounders and exclusion of age-related $\mathrm{CpG}$, differences were shown between AN individuals and controls. We initially investigated the differentially methylated regions (DMRs) in AN using a computational algorithm MethylKit with a stringent statistical cutoff of q-value (FDR adjusted p-value $<0.01$ ) and a minimum $25 \%$ change in methylation between AN patients and controls.

\section{Results}

This approach allowed us to identify a total of 153 DMRs in AN (Table 1). Of these, 53 DMRs were hypomethylated and 100 were hypermethylated, indicating that more genic regions tended to be methylated in AN individuals, similar to previous observations [9]. We further examined the genomic distribution of differentially methylated CpGs (DMCs). A total of 1812 DMC sites were identified in patients with AN compared to controls (Supplementary Table 1). Among the 1812 DMCs, 748 were hypomethylated, while 1065 were hypermethylated in the AN samples. Among these 1812 DMCs, 216 functional genic regions included at least 2 DMCs. Interestingly, when we compared our DMCs list with the short list of Steiger and colleagues, 32 genes were found in both lists (Supplementary Table 2) [9]. Moreover, among the 100 topranked differentially methylated positions identified in patients with major depressive disorder, six were also found in our study (GNG4, SLC39A12, CRTAC1, MYO7A, NTM and RMST), four of them playing a role in neurite extension and neurogenesis (GNG4, SLC39A12, NTM and RMST) [8].

To identify the molecular pathways and functions potentially influenced by methylation changes in AN, we performed GO term and KEGG pathway enrichment analyses of the genes closest to the identified DMRs (within the gene body or within $+/-10 \mathrm{~kb}$ of gene start/ end sites) using the DAVID bioinformatics resources 6.8 (https://david. ncifcrf.gov/). When "Disease" was used for categorization, there were 5 charts categories (with a significant $\mathrm{p}$ value with Benjamini correction) of DMRs, the more significant charts being waist-hip ratio ( $\mathrm{n}=9$ counts, Benjamini 5.2e-3), tobacco use disorder (37 counts, Benjamini 2.8e-3),

Table 1. Identification of differentially methylated regions (DMR) was performed using the predefined regions of Illumina TruSeq Methylcapture EPIC kit presenting at least $25 \%$ methylation difference between ANcases and controls (column Change) with a q-value (FDR corrected p-value) threshold of 0.01

\begin{tabular}{|c|c|c|c|c|c|c|}
\hline DMR & Chr & Nb Genes & Genes & $p$ value & $q$ value & Change \\
\hline chrX_114502896_114503051 & $\operatorname{chrX}$ & 0 & - & $2.22451 \mathrm{E}-15$ & $3.71584 \mathrm{E}-14$ & 59.78484933 \\
\hline chr14_54375093_54375328 & chr14 & 1 & AL138479. & $2.65207 \mathrm{E}-24$ & $1.18277 \mathrm{E}-22$ & 48.00682879 \\
\hline chr8_80036078_80036321 & chr8 & 0 & - & $2.44128 \mathrm{E}-36$ & $2.55548 \mathrm{E}-34$ & 46.08190008 \\
\hline chr11_79289117_79289197 & $\operatorname{chr} 11$ & 0 & - & $2.09678 \mathrm{E}-48$ & $3.89352 \mathrm{E}-46$ & 44.01889299 \\
\hline chr2_165342265_165342368 & $\operatorname{chr} 2$ & 1 & GRB14 & 5.17941E-11 & $4.44995 \mathrm{E}-10$ & 43.62139918 \\
\hline chr5_118811413_118811668 & chr5 & 2 & HSD17B4|snoU1 & $4.63731 \mathrm{E}-11$ & $4.01928 \mathrm{E}-10$ & 43.59922516 \\
\hline chr19_45113900_45114110 & chr19 & 2 & CEACAM22P|IGSF23 & $9.32156 \mathrm{E}-32$ & 7.39337E-30 & 40.63822929 \\
\hline chr12_39885082_39885307 & $\operatorname{chr} 12$ & 0 & - & $1.48466 \mathrm{E}-54$ & $3.57503 \mathrm{E}-52$ & 40.49521423 \\
\hline chr11_99564881_99564954 & $\operatorname{chr} 11$ & 1 & CNTN5 & $9.37129 \mathrm{E}-15$ & $1.42916 \mathrm{E}-13$ & 39.62334329 \\
\hline chr20_46228572_46228783 & chr20 & 1 & NCOA3 & 1.11712E-13 & $1.47266 \mathrm{E}-12$ & 39.56217898 \\
\hline chr12_9507242_9507307 & $\operatorname{chr} 12$ & 0 & - & $1.05726 \mathrm{E}-24$ & $4.90641 \mathrm{E}-23$ & 38.46786372 \\
\hline chr2_238213149_238213414 & chr2 & 0 & - & $1.58339 \mathrm{E}-06$ & $5.42033 \mathrm{E}-06$ & 37.58090232 \\
\hline chr9_88891303_88891317 & chr9 & 1 & ISCA1 & $1.08415 \mathrm{E}-06$ & $3.8617 \mathrm{E}-06$ & 37.31409719 \\
\hline chr7_134279040_134279370 & chr7 & 0 & - & 7.42127E-19 & $1.90865 \mathrm{E}-17$ & 36.8404498 \\
\hline chr11_125477842_125477944 & $\operatorname{chr} 11$ & 1 & STT3A & $1.37342 \mathrm{E}-52$ & $3.02928 \mathrm{E}-50$ & 36.14363165 \\
\hline chr4_85873367_85873551 & chr4 & 1 & WDFY3 & $8.771 \mathrm{E}-29$ & $5.54172 \mathrm{E}-27$ & 35.86458464 \\
\hline chr1_228243499_228243750 & chr1 & 1 & WNT3A & $7.15126 \mathrm{E}-21$ & $2.28614 \mathrm{E}-19$ & 35.584194 \\
\hline chr2_131046231_131046636 & $\mathrm{chr} 2$ & 5 & $\begin{array}{c}\text { MTND4P2 } \mid \text { MTND5P2 } \mid \text { MTND6P } \mid \text { AC068 } \\
137.1 \mid \text { AC068137.1 }\end{array}$ & $1.32673 \mathrm{E}-40$ & $1.75348 \mathrm{E}-38$ & 35.46103678 \\
\hline chr7_39723763_39723940 & chr7 & 1 & RALA & $2.01448 \mathrm{E}-11$ & $1.85339 \mathrm{E}-10$ & 34.0085943 \\
\hline chr6_132297421_132297621 & chr6 & 1 & RP11-69I8. & 0.001384883 & 0.002169711 & 33.37310303 \\
\hline chr3_108880309_108880502 & chr3 & 0 & - & 7.64309E-11 & $6.36725 \mathrm{E}-10$ & 33.06601128 \\
\hline chr1_66753890_66754101 & chr1 & 1 & PDE4B & 0.00182074 & 0.00275054 & 32.96104032 \\
\hline chr2_66654487_66654810 & $\operatorname{chr} 2$ & 2 & MEIS1-AS3|MEIS1 & $2.10022 \mathrm{E}-69$ & $7.72486 \mathrm{E}-67$ & 32.72071839 \\
\hline chr5_97764439_97764614 & $\operatorname{chr} 5$ & 0 & - & 0.000291866 & 0.000557559 & 32.43193392 \\
\hline chr9_115957266_115957508 & chr9 & 1 & FKBP15 & $1.69011 \mathrm{E}-15$ & $2.87555 \mathrm{E}-14$ & 32.29050602 \\
\hline chr15_50209373_50209588 & $\operatorname{chr} 15$ & 1 & ATP8B4 & $3.01371 \mathrm{E}-25$ & $1.45926 \mathrm{E}-23$ & 31.98261372 \\
\hline chr17_11608628_11608839 & $\operatorname{chr} 17$ & 1 & DNAH9 & 6.97091E-30 & $4.8207 \mathrm{E}-28$ & 31.79815222 \\
\hline chr4_3040168_3040507 & $\mathrm{chr} 4$ & 2 & GRK4|RNU6-204 & $7.3598 \mathrm{E}-14$ & $9.96918 \mathrm{E}-13$ & 31.65690685 \\
\hline chr6_88956453_88956584 & chr6 & 0 & - & $9.99041 \mathrm{E}-12$ & $9.68676 \mathrm{E}-11$ & 31.54201058 \\
\hline chr21_15436054_15437354 & $\operatorname{chr} 21$ & 3 & AP001347.|ANKRD20A18|RNA5SP48 & 0 & 0 & 31.16688815 \\
\hline chr3_49712243_49712522 & $\operatorname{chr} 3$ & 4 & BSN|APEH|MST1|AC099668. & $8.07314 \mathrm{E}-25$ & $3.79115 \mathrm{E}-23$ & 31.03180163 \\
\hline chr12_25264152_25264371 & $\operatorname{chr} 12$ & 2 & LRMP|CASC1 & $8.78331 \mathrm{E}-27$ & $4.8313 \mathrm{E}-25$ & 30.29058703 \\
\hline chrX_9121569_9121778 & $\operatorname{chrX}$ & 1 & FAM9B & 0.000426217 & 0.000777831 & 30.08088109 \\
\hline
\end{tabular}




\begin{tabular}{|c|c|c|c|c|c|c|}
\hline chr3_84932517_84932772 & chr3 & 2 & LINC00971|LINC02025 & $2.07283 \mathrm{E}-18$ & 5.07715E-17 & 29.94169954 \\
\hline chr7_123928236_123928461 & chr7 & 2 & RP5-921G16.|RP11-264K23. & $3.518 \mathrm{E}-31$ & $2.67311 \mathrm{E}-29$ & 29.91216787 \\
\hline chr9_106087270_106087465 & chr9 & 1 & LINC01492 & $8.36825 \mathrm{E}-19$ & $2.13729 \mathrm{E}-17$ & 29.78985686 \\
\hline chr5_120388958_120389191 & $\operatorname{chr} 5$ & 2 & AC008565.|CTD-2613O8. & $7.61105 \mathrm{E}-44$ & $1.17667 \mathrm{E}-41$ & 29.58350371 \\
\hline chr11_8927411_8927650 & chr11 & 2 & ST5|AKIP1 & $4.49366 \mathrm{E}-10$ & 3.25802E-09 & 29.52623011 \\
\hline chr6_159572123_159572413 & chr6 & 0 & - & $7.42108 \mathrm{E}-39$ & $8.88692 \mathrm{E}-37$ & 29.31934038 \\
\hline chr22_49625975_49626187 & $\operatorname{chr} 22$ & 0 & - & $2.92003 \mathrm{E}-11$ & $2.61571 \mathrm{E}-10$ & 29.27879223 \\
\hline chr12_9776968_9777167 & $\operatorname{chr} 12$ & 2 & LOC374443|RNU6-700 & $1.12651 \mathrm{E}-09$ & 7.58502E-09 & 29.08923252 \\
\hline chr3_170332310_170332590 & chr3 & 1 & SLC7A14-AS1 & $1.8717 \mathrm{E}-08$ & $9.81278 \mathrm{E}-08$ & 29.01098901 \\
\hline chr4_77986307_77986536 & chr4 & 1 & CCNI & $3.91084 \mathrm{E}-14$ & $5.48555 \mathrm{E}-13$ & 28.97085991 \\
\hline chr22_42176013_42176129 & chr22 & 1 & MEI1 & 0.000206038 & 0.000410706 & 28.81962113 \\
\hline chr10_25240859_25240994 & chr10 & 2 & PRTFDC1|RP11-165A20. & $6.26124 \mathrm{E}-28$ & $3.71906 \mathrm{E}-26$ & 28.60587752 \\
\hline chr3_79966821_79966915 & chr3 & 0 & - & $1.15638 \mathrm{E}-10$ & $9.34284 \mathrm{E}-10$ & 28.60217066 \\
\hline chr3_45649141_45649463 & $\operatorname{chr} 3$ & 1 & LIMD1 & $6.28165 \mathrm{E}-62$ & $1.8899 \mathrm{E}-59$ & 28.50314686 \\
\hline chr6_25166613_25166879 & chr6 & 1 & СМАНР & $2.17351 \mathrm{E}-96$ & $1.46968 \mathrm{E}-93$ & 28.37986243 \\
\hline chr3_152213629_152213846 & chr3 & 1 & RP11-362A9. & $6.8342 \mathrm{E}-05$ & 0.000155335 & 28.26746276 \\
\hline chr11_59836664_59836958 & chr11 & 2 & MS4A3|RP11-736I10. & $3.63343 \mathrm{E}-09$ & 2.21257E- 08 & 28.0516934 \\
\hline chr20_14124320_14124383 & chr20 & 1 & MACROD2 & $1.40341 \mathrm{E}-20$ & 4.35847E-19 & 28.02413196 \\
\hline chr22_32728242_32728474 & chr22 & 1 & RP1-149A16.1 & $8.85421 \mathrm{E}-11$ & 7.29047E-10 & 27.91646442 \\
\hline chr2_183106034_183106226 & chr2 & 1 & PDE1A & $9.65506 \mathrm{E}-09$ & $5.3791 \mathrm{E}-08$ & 27.85254866 \\
\hline chr6_118401762_118401938 & chr6 & 2 & SLC35F1|LOC105377967 & 4.64459E-19 & $1.22408 \mathrm{E}-17$ & 27.79487179 \\
\hline chr10_91369814_91369992 & chr10 & 1 & PANK1 & $1.57036 \mathrm{E}-21$ & $5.3785 \mathrm{E}-20$ & 27.77056277 \\
\hline chr12_49121182_49121257 & $\operatorname{chr} 12$ & 1 & TEX49 & $8.24722 \mathrm{E}-13$ & $9.54004 \mathrm{E}-12$ & 27.60425962 \\
\hline chr5_95362159_95362388 & chr5 & 1 & LOC101929710 & $4.61645 \mathrm{E}-19$ & $1.21738 \mathrm{E}-17$ & 27.51574796 \\
\hline chr17_16935456_16935522 & $\operatorname{chr} 17$ & 0 & - & $4.58885 \mathrm{E}-09$ & 2.74027E-08 & 27.46293683 \\
\hline chr3_42093299_42093629 & chr3 & 2 & TRAK1|RP11-193I22. & $6.20321 \mathrm{E}-37$ & 6.69223E-35 & 27.41943731 \\
\hline chr3_149867507_149867632 & chr3 & 1 & LOC105374313 & $3.07268 \mathrm{E}-11$ & $2.74327 \mathrm{E}-10$ & 27.29693742 \\
\hline chrX_43503885_43504133 & $\operatorname{chrX}$ & 0 & - & 4.40393E-09 & 2.64009E-08 & 27.18926273 \\
\hline chr14_77035752_77035960 & chr14 & 1 & RP11-18707. & $6.2288 \mathrm{E}-12$ & $6.23127 \mathrm{E}-11$ & 27.15821169 \\
\hline chr10_52771069_52771259 & chr10 & 1 & PRKG1 & $3.87096 \mathrm{E}-16$ & $7.14936 \mathrm{E}-15$ & 27.04932754 \\
\hline chr2_38055293_38055466 & chr2 & 1 & LINC00211 & $7.48568 \mathrm{E}-28$ & $4.4228 \mathrm{E}-26$ & 27.03032591 \\
\hline chr1_108245511_108245769 & chr1 & 1 & VAV3 & $3.17027 \mathrm{E}-12$ & $3.3165 \mathrm{E}-11$ & 26.94594044 \\
\hline chr11_132891631_132891819 & chr11 & 1 & OPCML & 4.6077E-05 & 0.000109674 & 26.80024478 \\
\hline chr8_8538073_8538307 & chr8 & 0 & - & $6.60262 \mathrm{E}-14$ & 8.99085E-13 & 26.64249158 \\
\hline chr8_107757803_107757980 & chr8 & 1 & OXR1 & 0.000716855 & 0.001224528 & 26.62186488 \\
\hline chr21_36250641_36250940 & chr21 & 2 & LOC100506403|RUNX1 & $3.11176 \mathrm{E}-25$ & $1.50565 \mathrm{E}-23$ & 26.55673748 \\
\hline chr9_14910433_14910528 & chr9 & 1 & FREM1 & $8.33159 \mathrm{E}-21$ & 2.64892E-19 & 26.53151371 \\
\hline chr3_10851357_10851586 & chr3 & 1 & SLC6A11 & 0.004410223 & 0.005890646 & 26.40737551 \\
\hline chr9_73216788_73217020 & $\operatorname{chr} 9$ & 1 & TRPM3 & $3.0921 \mathrm{E}-15$ & 5.06212E-14 & 26.40133464 \\
\hline chr17_77906666_77906803 & chr17 & 3 & LINC01979|LINC01978|TBC1D16 & $3.90756 \mathrm{E}-08$ & $1.9197 \mathrm{E}-07$ & 26.38634157 \\
\hline chr15_101507155_101507328 & chr15 & 1 & LRRK1 & 5.16927E-09 & $3.05155 \mathrm{E}-08$ & 26.37814064 \\
\hline chr10_87988149_87988359 & chr10 & 1 & GRID1 & 0.003497861 & 0.004828383 & 26.35618749 \\
\hline chr13_46978725_46978934 & chr13 & 2 & RUBCNL|RNU6-68 & $2.29746 \mathrm{E}-12$ & $2.46329 \mathrm{E}-11$ & 26.04182226 \\
\hline chrX_149197068_149197285 & $\operatorname{chrX}$ & 1 & LINC00894 & $2.44211 \mathrm{E}-07$ & $1.01031 \mathrm{E}-06$ & 26.00779648 \\
\hline chr16_66453623_66453908 & chr16 & 2 & LINC00920|BEAN1 & 3.68957E-06 & 1.15579E-05 & 25.85630262 \\
\hline chr1_165186330_165186523 & chr1 & 3 & LMX1A|RP11-38C18.|RP11-38C18. & 0.000275984 & 0.000530947 & 25.72822521 \\
\hline chr12_6662473_6662654 & $\operatorname{chr} 12$ & 3 & IFFO1|RP5-940J5.|NOP2 & $9.5204 \mathrm{E}-29$ & $6.00387 \mathrm{E}-27$ & 25.71991419 \\
\hline chr4_129308317_129308548 & chr4 & 1 & LINC02615 & $2.02201 \mathrm{E}-09$ & 1.29361E-08 & 25.68972744 \\
\hline chr4_102756899_102757268 & chr4 & 1 & BANK1 & $8.27941 \mathrm{E}-46$ & $1.39081 \mathrm{E}-43$ & 25.66642434 \\
\hline chr6_101852800_101852969 & chr6 & 1 & GRIK2 & $1.14283 \mathrm{E}-19$ & 3.23959E-18 & 25.50742035 \\
\hline chr7_147500652_147500831 & chr7 & 1 & CNTNAP2 & 4.69478E-06 & $1.43535 \mathrm{E}-05$ & 25.47112462 \\
\hline chr2_130683180_130683508 & chr2 & 2 & PLAC9P1|LINC01856 & $2.6716 \mathrm{E}-16$ & $5.03565 \mathrm{E}-15$ & 25.46823547 \\
\hline chr3_69157750_69157970 & chr3 & 2 & ARL6IP5|LMOD3 & $6.39788 \mathrm{E}-06$ & $1.89017 \mathrm{E}-05$ & 25.46542143 \\
\hline chr12_104676677_104676883 & $\operatorname{chr} 12$ & 2 & TXNRD1|RP11-818F20. & 4.32249E-15 & $6.92567 \mathrm{E}-14$ & 25.4273867 \\
\hline chr9_4375797_4376098 & chr9 & 1 & AL162419. & $3.07166 \mathrm{E}-06$ & 9.81114E-06 & 25.41113806 \\
\hline chr6_55377549_55377764 & chr6 & 1 & HMGCLL1 & $4.38355 \mathrm{E}-45$ & 7.21874E-43 & 25.36445879 \\
\hline chr8_80816076_80816210 & chr8 & 0 & - & $2.50289 \mathrm{E}-06$ & $8.16528 \mathrm{E}-06$ & 25.34385329 \\
\hline chr5_171810643_171810847 & chr5 & 1 & SH3PXD2B & $2.88223 \mathrm{E}-10$ & $2.16508 \mathrm{E}-09$ & 25.2488391 \\
\hline chr2_66648614_66648933 & chr2 & 1 & MEIS1-AS3 & $1.28929 \mathrm{E}-22$ & 4.90242E-21 & 25.21383356 \\
\hline chr14_96583490_96583604 & chr14 & 0 & - & 0.003078364 & 0.004325989 & 25.19935377 \\
\hline chr22_16864741_16864893 & $\operatorname{chr} 22$ & 1 & ABCD1P & 0.002259786 & 0.003313591 & 25.19298246 \\
\hline chr10_90483503_90483709 & $\operatorname{chr} 10$ & 2 & LIPK|KRT8P3 & $3.47526 \mathrm{E}-44$ & $5.45461 \mathrm{E}-42$ & 25.15294597 \\
\hline
\end{tabular}




\begin{tabular}{|c|c|c|c|c|c|c|}
\hline chr6_7699982_7700169 & chr6 & 0 & - & $1.47903 \mathrm{E}-12$ & $1.64125 \mathrm{E}-11$ & 25.12462285 \\
\hline chr3_195501977_195502276 & chr3 & 1 & MUC4 & $1.31243 \mathrm{E}-47$ & $2.3649 \mathrm{E}-45$ & 25.08813062 \\
\hline chr18_29303346_29303538 & $\operatorname{chr} 18$ & 3 & RN7SKP4|LRRC37A7|RP11-549B18. & $1.62011 \mathrm{E}-27$ & $9.3655 \mathrm{E}-26$ & 25.03570419 \\
\hline chr3_95424692_95425326 & chr3 & 0 & - & $3.3968 \mathrm{E}-14$ & $4.80683 \mathrm{E}-13$ & 25.03516155 \\
\hline chr3_180102299_180102384 & chr3 & 0 & - & $9.13491 \mathrm{E}-07$ & $3.3066 \mathrm{E}-06$ & 25.01885857 \\
\hline chr22_35587422_35587713 & $\operatorname{chr} 22$ & 2 & LINC01399|COX7BP & $1.08406 \mathrm{E}-10$ & $8.79565 \mathrm{E}-10$ & -25.01400968 \\
\hline chr20_36132841_36133069 & $\operatorname{chr} 20$ & 1 & BLCAP & $1.55091 \mathrm{E}-18$ & $3.84306 \mathrm{E}-17$ & -25.14666861 \\
\hline chr19_47082930_47083060 & $\operatorname{chr} 19$ & 2 & PPP5D1|AC011551. & $6.29188 \mathrm{E}-21$ & $2.02349 \mathrm{E}-19$ & -25.24712874 \\
\hline chr19_49572906_49573157 & $\operatorname{chr} 19$ & 4 & $\begin{array}{c}\text { NTF4|CTB-60B18.1|CTB- } \\
\text { 60B18.1|KCNA7 }\end{array}$ & 5.89203E-07 & $2.23171 \mathrm{E}-06$ & -25.34019384 \\
\hline chr3_11489044_11489192 & $\operatorname{chr} 3$ & 1 & ATG7 & $7.90562 \mathrm{E}-10$ & $5.47842 \mathrm{E}-09$ & -25.40198715 \\
\hline chr17_75631717_75631979 & chr17 & 0 & - & $1.84137 \mathrm{E}-05$ & $4.85349 \mathrm{E}-05$ & -25.53803605 \\
\hline chr1_19176711_19177105 & chr1 & 2 & TAS1R2|RP13-279N23. & 0.000375134 & 0.000695164 & -25.58525242 \\
\hline chr11_5840963_5841191 & chr11 & 2 & TRIM5|OR52N2 & $2.88714 \mathrm{E}-66$ & $9.68772 \mathrm{E}-64$ & -25.69185678 \\
\hline chr2_55378165_55378300 & chr2 & 0 & - & $9.89458 \mathrm{E}-19$ & $2.51065 \mathrm{E}-17$ & -25.78431373 \\
\hline chr13_19315890_19316198 & $\operatorname{chr} 13$ & 3 & ZNF965|LINC00417|CYP4F34 & $8.90044 \mathrm{E}-14$ & $1.19079 \mathrm{E}-12$ & -25.87364383 \\
\hline chr18_61669993_61670289 & $\operatorname{chr} 18$ & 1 & SERPINB8 & $8.41432 \mathrm{E}-63$ & $2.5838 \mathrm{E}-60$ & -26.04262437 \\
\hline chr20_32885922_32886673 & chr20 & 1 & AHCY & $2.8332 \mathrm{E}-44$ & $4.47838 \mathrm{E}-42$ & -26.45197851 \\
\hline chr6_106252261_106252558 & chr6 & 0 & - & $6.2211 \mathrm{E}-107$ & $4.9875 \mathrm{E}-104$ & -26.54101932 \\
\hline chr2_25383849_25384399 & chr2 & 3 & EFR3B|RP11-509E16|POMC & $4.25503 \mathrm{E}-85$ & $2.26062 \mathrm{E}-82$ & -26.70759651 \\
\hline chr4_131633369_131633569 & $\operatorname{chr} 4$ & 0 & - & $1.21778 \mathrm{E}-09$ & $8.14225 \mathrm{E}-09$ & -26.8218303 \\
\hline chr4_26789871_26790007 & $\operatorname{chr} 4$ & 0 & - & $9.76527 \mathrm{E}-06$ & $2.75795 \mathrm{E}-05$ & -27.15229384 \\
\hline chr1_55370080_55370265 & chr1 & 0 & - & $1.33489 \mathrm{E}-60$ & $3.89366 \mathrm{E}-58$ & -27.2302102 \\
\hline chr19_45619267_45619497 & chr19 & 2 & MARK4|PPP1R37 & $3.61011 \mathrm{E}-48$ & $6.62101 \mathrm{E}-46$ & -27.29034787 \\
\hline chr16_33588844_33589010 & $\operatorname{chr} 16$ & 1 & ENPP7P13 & $8.03476 \mathrm{E}-26$ & $4.0933 \mathrm{E}-24$ & -27.38040136 \\
\hline chr4_15907926_15908145 & chr4 & 0 & - & $2.8442 \mathrm{E}-50$ & $5.76083 \mathrm{E}-48$ & -27.44818039 \\
\hline chr10_67155111_67155236 & $\operatorname{chr} 10$ & 0 & - & $4.83108 \mathrm{E}-11$ & 4.17264E-10 & -27.80333564 \\
\hline chr17_4278292_4278786 & $\operatorname{chr} 17$ & 1 & UBE2G1 & $9.00939 \mathrm{E}-24$ & $3.82074 \mathrm{E}-22$ & -27.82640422 \\
\hline chr11_116344108_116344147 & chr11 & 0 & - & 0.000326633 & 0.000615342 & -27.8388828 \\
\hline chr19_34245878_34246069 & chr19 & 1 & CHST8 & $6.69284 \mathrm{E}-06$ & $1.96741 \mathrm{E}-05$ & -27.92649973 \\
\hline chr17_16864290_16864524 & $\operatorname{chr} 17$ & 1 & TNFRSF13B & $1.26001 \mathrm{E}-12$ & $1.41214 \mathrm{E}-11$ & -28.2842299 \\
\hline chr11_82354426_82354705 & chr11 & 2 & MIR4300HG|RP11-179A16. & $6.09835 \mathrm{E}-07$ & $2.30172 \mathrm{E}-06$ & -29.18358119 \\
\hline chr5_153676389_153676633 & chr5 & 1 & GALNT10 & $1.43406 \mathrm{E}-68$ & $5.14737 \mathrm{E}-66$ & -29.70725527 \\
\hline chr10_134778418_134779164 & chr10 & 3 & LINC01166|LINC01167|LINC01168 & $5.1864 \mathrm{E}-178$ & $1.2399 \mathrm{E}-174$ & -29.73911375 \\
\hline chr8_36995835_36995986 & chr8 & 1 & MIR1268A & $3.40507 \mathrm{E}-09$ & $2.0846 \mathrm{E}-08$ & -29.75107115 \\
\hline chr2_213316451_213316721 & chr2 & 1 & ERBB4 & $3.25312 \mathrm{E}-05$ & $8.05895 \mathrm{E}-05$ & -30.06050559 \\
\hline chr3_105601223_105601548 & chr3 & 0 & - & $3.99011 \mathrm{E}-26$ & $2.08106 \mathrm{E}-24$ & -30.45696192 \\
\hline chr2_130693963_130694229 & chr2 & 3 & PLAC9P1|LINC01856|AC079776. & $1.8989 \mathrm{E}-114$ & $1.6726 \mathrm{E}-111$ & -30.54653298 \\
\hline chr11_110986763_110986975 & chr11 & 1 & RP11-89C3. & 0 & 0 & -30.61868687 \\
\hline chr4_184296182_184296485 & chr4 & 2 & RP11-451F20.|AC093844. & $3.2812 \mathrm{E}-82$ & $1.64531 \mathrm{E}-79$ & -30.6235367 \\
\hline chr6_21452696_21452935 & chr6 & 0 & - & $4.66098 \mathrm{E}-09$ & $2.77827 \mathrm{E}-08$ & -30.7652865 \\
\hline chr14_56669107_56669307 & $\operatorname{chr} 14$ & 1 & PELI2 & 0.005344129 & 0.006946025 & -31.10639369 \\
\hline chr13_25141770_25142091 & $\operatorname{chr} 13$ & 3 & TPTE2P6|PSPC1P|RP11-556N21. & $2.11697 \mathrm{E}-25$ & $1.04092 \mathrm{E}-23$ & -31.10709988 \\
\hline chr2_238796929_238797268 & chr2 & 1 & RAMP1 & $9.04001 \mathrm{E}-24$ & $3.83251 \mathrm{E}-22$ & -31.35072812 \\
\hline chr17_6901611_6901751 & $\operatorname{chr} 17$ & 4 & $\begin{array}{l}\text { ALOX12-AS1|RP11-589P10.|RP11- } \\
\text { 589P10.|ALOX12 }\end{array}$ & 0.00014541 & 0.000302279 & -31.60823695 \\
\hline chr15_79299672_79299707 & chr15 & 1 & RASGRF1 & 0.004476538 & 0.005967375 & -32.72230999 \\
\hline chr1_223353214_223353363 & chr1 & 1 & RP11-239E10 & $7.13359 \mathrm{E}-18$ & $1.64468 \mathrm{E}-16$ & -33.00889209 \\
\hline chr1_113221602_113221746 & chr1 & 2 & CAPZA1|MOV10 & $8.44636 \mathrm{E}-34$ & $7.62526 \mathrm{E}-32$ & -33.03827068 \\
\hline chr19_21860552_21861267 & chr19 & 1 & RP11-420K14. & $1.3469 \mathrm{E}-284$ & $1.127 \mathrm{E}-280$ & -33.08420719 \\
\hline chr2_145505959_145506291 & chr2 & 2 & TEX41|AC023128. & $2.36911 \mathrm{E}-23$ & $9.63501 \mathrm{E}-22$ & -33.13077409 \\
\hline chr21_29565178_29565285 & chr21 & 1 & LINC01695 & 0.000196292 & 0.000393653 & -33.4915241 \\
\hline chr22_47442504_47442671 & $\operatorname{chr} 22$ & 1 & TBC1D22A & $2.44869 \mathrm{E}-31$ & $1.87658 \mathrm{E}-29$ & -34.87001855 \\
\hline chr8_119449323_119449547 & chr8 & 1 & SAMD12 & $2.1869 \mathrm{E}-06$ & 7.23312E-06 & -36.3138769 \\
\hline chr3_6706652_6706860 & chr3 & 2 & AC069277.|GRM7-AS3 & 0.00028562 & 0.000547251 & -39.63810451 \\
\hline chr8_129286309_129286584 & chr8 & 0 & - & $3.30084 \mathrm{E}-09$ & $2.02608 \mathrm{E}-08$ & -39.75752508 \\
\hline chr12_64067778_64067878 & $\operatorname{chr} 12$ & 3 & MIR548Z|DPY19L2|RP11-415I12. & 0.000389652 & 0.000718873 & -40.8170893 \\
\hline chr15_71187717_71187868 & chr15 & 2 & LRRC49|THAP10 & 0.004522921 & 0.006020138 & -42.45762712 \\
\hline chr3_115099771_115100023 & chr3 & 0 & - & 0.002555418 & 0.003683188 & -42.60471914 \\
\hline chr17_57187586_57187924 & chr17 & 3 & TRIM37|AC099850.|SKA2 & $2.39899 \mathrm{E}-60$ & $6.93704 \mathrm{E}-58$ & -46.21784352 \\
\hline
\end{tabular}


chemdependency ( $\mathrm{n}=40$ counts, Benjamini 1.2e-3), metabolic $(\mathrm{n}=49$, Benjamini 3.1e-3) and neurological (32 counts, Benjamini 3.8e-3).

We next focused on the 153 DMRs, the String network analysis software identified a subnetwork, related to MAPK signaling pathway (hs04010, FDR 0.0026), PI3K-Akt signaling pathway (hsa04151, FDR 0.0232) and neurotrophin signaling pathway (hsa04550, FDR 0.0251)(https://string-db.org/cgi/network.pl?taskId=07ri9Goe1SzI) (Figure 1).

\section{Discussion}

To the best of our knowledge, the present study presents the first genome-wide DNA methylation profiling of 6 male AN patients, using a high-throughput DNA methylation sequencing covering a large number of $\mathrm{CpG}$ sites on the human genome. Patients affected with AN restrictive type showed many differentially methylated sites, with significant between group differences corresponding to genes implicated in metabolic and nutritional status, psychiatric status and immune function. When we compared our DMCs list with the short list of Steiger and colleagues obtained in female AN patients, 32 genes were found in both lists [9]. Moreover, because the interaction between depression and anorexia nervosa was significant, we also identified six genes previously found in patients with major depressive disorder [8]. Taking into account that our approach was based on genome-wide

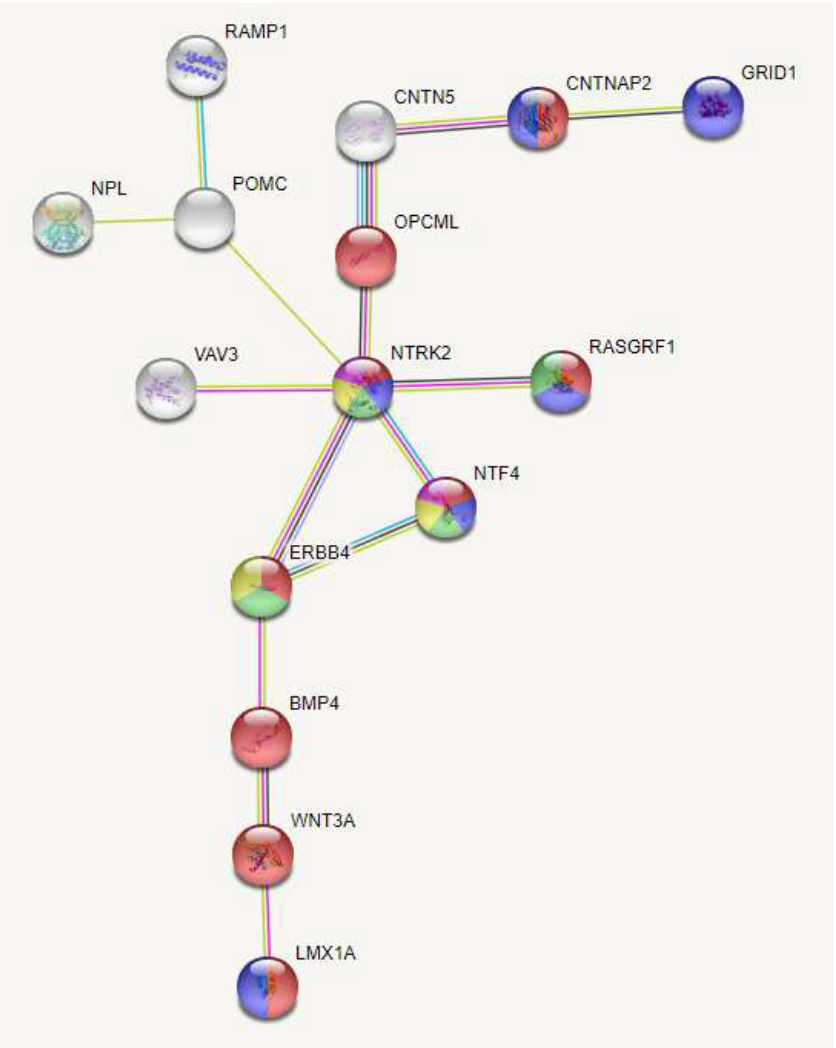

Figure 1. Protein subnetwork of the identified proteins constructed with the STRING software for enrichment analysis of proteins (PPI enrichment value 7.04e-08) showing a dysmethylation status (15 DMRs among the 119 identified DMRs including at least one coding or non-coding genes). Each node represents a protein entity. Gene Ontology identified functional enrichments for several biological process including neuron differentiation (GO:0030182, FDR 8.15e-06) and behavior (GO:0007610, FDR 0.00025)(in black), and KEGG pathways identified enrichments for several pathways including MAPK signaling pathway (hs04010, FDR 0.0026)(grey square), PI3K-Akt signaling pathway (hsa04151, FDR 0.0232)(white square) and Neurotrophin signaling pathway (hsa04550, FDR 0.0251) (grey triangle)(https://string-db.org/cgi/network.pl?taskId=07ri9Goe1SzI) methylation sequencing, we also identified additional candidate genes involved in psychiatric disorders (GRID1, NAALADL2-AS3, PDZD, CEP85L, GRIK3, SLC7A14), in metabolism regulation (lipids, ZFP36L1, CERK, ACSF3, EEPD1; glucose, KCNA7), and finally, in addiction (GDE1, CCKAR, PDYN). Moreover, we identified few genes previously associated with anorexia nervosa in genome-wide association studies (VGLL4, GRID1, WWOX, CAMK1D, SORCS2) (https://www.ebi. ac.uk/gwas/search?query=anorexia\%20nervosa). Interestingly, the String network analysis software revealed a subnetwork related to neurotrophin signaling pathway (Figure 1). The reported data, in addition to the previous reported findings for BDNF, NTRK2 and $N T R K 3$, point again neurotrophin signaling genes as key regulators of eating behavior.

To conclude, our data replicates several results concerning several target genes such as PRKAG2, RPTOR, and ICAM5 previously discussed [9], and identified novel signaling pathways involving PI3K-Akt and neurotrophin signaling pathway disturbed in anorexia nervosa. Future replication of findings in male AN patients will be a determinant.

\section{Compliance with ethical standards}

\section{Funding}

This study was supported by Institut National de la Recherche Médicale (INSERM) and by Fondation Maladies Rares (program High throughput sequencing and rare disease, number \#11632).

\section{Conflicts of interest}

The authors declare that they have no conflicts of interest.

\section{Ethical approval}

All procédures performed in this study were in accordance with the ethical standards of our national research committee and with the 1964 Helsinki declaration and its later amendments. Informed written consent was obtained from all patients and parents included in the study.

\section{Acknowledgements}

We thank the families for their enthusiastic participation and all physicians from the different medical and psychiatric centers.

\section{References}

1. Bulik CM, Sullivan PF, Tozzi F, Furberg H, Lichtenstein P, et al. (2006) Prevalence, heritability, and prospective risk factors for anorexia nervosa. Arch Gen Psychiatry 63: 305-312. [Crossref]

2. Wang K, Zhang H, Bloss CS, Duvvuri V, Kaye W, Schork NJ, et al. (2011) A genomewide association study on common SNPs and rare CNVs in anorexia nervosa. Mol Psychiatry 16: 949-959. [Crossref]

3. Boraska V, Franklin CS, Floyd JA, Thornton LM, Huckins LM, et al. (2014) A genome-wide association study of anorexia nervosa. Mol Psychiatry 19: 1085-1094. [Crossref]

4. Duncan L, Yilmaz Z, Gaspar H, Walters R, GoldsteinJ, Anttila V, et al. (2017) Significant locus and metabolic genetic correlations revealed in genome-wide association study of anorexia nervosa. Am J Psychiatry 174: 850-858. [Crossref]

5. Huckins LM, Hatzikotoulas K, Southam L, Thornton LM, Steinberg J, Aguilera-McKay F, et al. (2018) Revealing the complex genetic architecture of obsessive-compulsive disorder using meta-analysis. Mol Psychiatry 23: 1181-1188. [Crossref]

6. Thornton LM, Welch E, Munn-Chernoff MA, Lichtenstein P, Bulik CM (2016) Anorexia nervosa, major depression, and suicide attempts: shared genetic factors. Suicide Life Threat Behav 46: 525-534. [Crossref] 
7. Wade TD, Bulik CM, Neale M, Kendler KS (2000) Anorexia nervosa and major depression: shared genetic and environmental risk factors. Am J Psychiatry 157: 469471. [Crossref]

8. Shimada M, Otowa T, Miyagawa T, Umekage T, Kawamura Y, Bundo M, et al. (2018) Anepigenome-wide methylation study of healthy individuals with or without depressive symptoms. J Hum Genet 63: 319-326. [Crossref]
9. Steiger H, Booij L, Kahan, McGregor K, Thaler L, Fletcher E, et al. (2019) A longitudinal,epigenome-wide study of DNA methylation in anorexia nervosa: results in actively ill, partially weight-restored, long-term remitted and non-eatingdisorderedwomen. $J$ Psychiatry Neurosci 44: 1-9. [Crossref]

10. Akalin A, Kormaksson M, Li S, Garrett-Bakelman FE, Figueroa ME, Melnick A, et al (2012) MethylKit: a comprehensive R package for the analysis of genome-wideDNA methylation profiles. Genome Biol 13: 80-87. [Crossref]

Copyright: (2019 Kim A. This is an open-access article distributed under the terms of the Creative Commons Attribution License, which permits unrestricted use, distribution, and reproduction in any medium, provided the original author and source are credited. 